\title{
Impact of Obesity and Heavy Alcohol Consumption on Hepatocellular Carcinoma Development after HCV Eradication with Antivirals
}

\author{
Tatsuya Minami ${ }^{a}$ Ryosuke Tateishi ${ }^{a}$ Naoto Fujiwara ${ }^{a}$ Ryo Nakagomi ${ }^{a}$

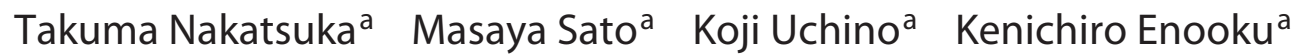

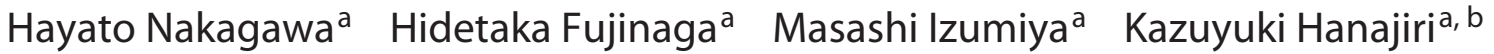 \\ Yoshinari Asaokaa,c Yuji Kondo ${ }^{a, d}$ Yasuo Tanaka ${ }^{a}$ Motoyuki Otsuka ${ }^{a}$ \\ Takamasa Ohki $^{\text {e }}$ Masahiro Arai ${ }^{f}$ Atsushi Tanaka ${ }^{c}$ Kiyomi Yasudag \\ Hideaki Miurah $^{\text {Itsuro Ogata }}{ }^{i}$ Toshiro Kamoshidaj Kazuaki Inoue ${ }^{k}$ \\ Yukihiro Koike Masatoshi Akamatsu $^{m}$ Hiroshi Mitsui ${ }^{\text {n }}$ Hajime Fujie $^{\circ}$ \\ Keiji Ogura $^{p}$ Hideo Yoshidaq Tomonori Wadab Kiyohiko Kurair \\ Hisato Maekawa ${ }^{\mathrm{s}}$ Shuntaro Obi ${ }^{\mathrm{d}}$ Takuma Teratani $^{\mathrm{t}}$ Naohiko Masaki ${ }^{\mathrm{u}}$ \\ Kayo Nagashimav Takashi Ishikawaw Naoya Kato ${ }^{a}$ Kyoji Moriya ${ }^{a}$ \\ Hiroshi Yotsuyanagi ${ }^{x}$ Kazuhiko Koikea SMART-C Investigators
}

\begin{abstract}
aDepartment of Gastroenterology, Graduate School of Medicine, The University of Tokyo, Tokyo, Japan; ${ }^{b}$ Department of Gastroenterology, Sanraku Hospital, Tokyo, Japan; 'Department of Medicine, Teikyo University School of Medicine, Tokyo, Japan; 'Department of Gastroenterology and Hepatology, Kyoundo Hospital, Tokyo, Japan; ${ }^{e}$ Department of Gastroenterology, Mitsui Memorial Hospital, Tokyo, Japan; 'Department of Gastroenterology, Toshiba General Hospital, Toshiba, Japan; 9Department of Gastroenterology, Kiyokawa Hospital, Tokyo, Japan; hDepartment of Gastroenterology, Tokyo Yamate Medical Center, Tokyo, Japan; 'Department of Gastroenterology, Kawakita General Hospital, Tokyo, Japan; jDepartment of Gastroenterology, Hitachi General Hospital, Hitachi, Japan; ${ }^{k}$ Department of Gastroenterology, Showa University Fujigaoka Hospital, Yokohama, Japan; 'Department of Gastroenterology, Kanto Central Hospital

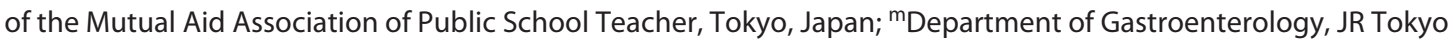
General Hospital, Tokyo, Japan; ${ }^{\mathrm{n} D e p a r t m e n t}$ of Gastroenterology, Tokyo Teishin Hospital, Tokyo, Japan; ${ }^{\circ}$ Department of Gastroenterology, Tokyo Shinjuku Medical Center, Tokyo, Japan; ${ }^{P}$ Department of Gastroenterology, Tokyo Metropolitan Police Hospital, Tokyo, Japan; ' Department of Gastroenterology, Japanese Red Cross Medical Center, Tokyo, Japan; 'Kurai Kiyohiko Medical Clinic, Utsunomiya, Japan; 's Department of Gastroenterology and Hepatology, Tokyo Takanawa Hospital, Tokyo, Japan; 'Department of Hepato-Biliary-Pancreatic medicine, NTT Medical Center Tokyo, Tokyo, Japan; "Clinical Laboratory Department, Center Hospital of the National Center for Global Health and Medicine, Tokyo, Japan; vDepartment of Gastroenterology, National Disaster Medical Center, Tachikawa, Japan; wMarunouchi Clinic, Tokyo, Japan; ${ }^{x}$ Division of Infectious Disease and Applied Immunology, The Institute of Medical Science Research Hospital, The University of Tokyo, Tokyo, Japan
\end{abstract}

T.M. and R.T. have equally contributed to this work.

karger@karger.com www.karger.com/lic

Karger $\stackrel{\text { ' }}{5}$

GOPEN ACCESS
(C) 2021 The Author(s)

Published by S. Karger AG, Basel

This is an Open Access article licensed under the Creative Commons Attribution-NonCommercial-4.0 International License (CC BY-NC) (http://www.karger.com/Services/OpenAccessLicense), applicable to the online version of the article only. Usage and distribution for commercial purposes requires written permission.
Correspondence to:

Ryosuke Tateishi, tateishi-tky@umin.ac.jp 


\section{Keywords}

Hepatitis C · Direct-acting antivirals · Hepatocellular

carcinoma $\cdot$ Obesity

\section{Abstract}

Background and Aims: It remains unclear whether obesity increases the risk of hepatocellular carcinoma (HCC) in patients with chronic hepatitis $C$ who achieved a sustained virological response (SVR) with antiviral therapy. Methods: In this multicenter cohort study, we enrolled patients with chronic hepatitis $C$ who achieved SVR with interferon (IFN)based therapy (IFN group) or direct-acting antiviral (DAA) therapy (DAA group) between January 1, 1990, and December 31, 2018. The patients underwent regular surveillance for $\mathrm{HCC}$. Cumulative incidence of and the risk factors for HCC development after SVR were assessed using the Kaplan-Meier method and Cox proportional hazard regression analysis, respectively. Results: Among 2,055 patients (840 in the IFN group and 1,215 in the DAA group), 75 developed HCC (41 in the IFN group and 34 in the DAA group) during the mean observation period of 4.1 years. The incidence rates of $\mathrm{HCC}$ at 1, 2, and 3 years were 1.2, 1.9, and 3.0\%, respectively. Multivariate analysis revealed that in addition to older age, lower albumin level, lower platelet count, higher alpha-fetoprotein level, and absence of dyslipidemia, obesity (body mass index $\geq 25 \mathrm{~kg} / \mathrm{m}^{2}$ ) and heavy alcohol consumption ( $\geq 60 \mathrm{~g} /$ day) were independent risk factors for HCC development, with adjusted hazard ratio (HR) of 2.53 (95\% confidence interval [Cl]: 1.51-4.25) and 2.56 (95\% Cl: 1.14-5.75), respectively. The adjusted HR was not significant between the 2 groups (DAA vs. IFN; HR 1.19, 95\% Cl: 0.61-2.33). Conclusions: Obesity and heavy alcohol consumption increased the risk of HCC development after SVR.

(c) 2021 The Author(s)
Published by S. Karger AG, Basel

\section{Introduction}

Hepatitis $\mathrm{C}$ virus (HCV) infection is a major global health burden, with an estimated prevalence of $1 \%$ worldwide [1], and it is a leading risk factor for hepatocellular carcinoma (HCC) [2-4]. Previously, interferon (IFN)based therapy was the standard of care for chronic HCV infection. Currently, with the emergence of direct-acting antivirals (DAA), sustained viral response (SVR) can be achieved at a rate of nearly $100 \%$, with favorable tolerability even in patients with advanced liver disease [5-8].

Now that who to treat is no longer a controversial issue $[9,10]$, and the vast majority of patients with chronic hep- atitis C would achieve SVR sooner or later, a remaining primary concern is the development of HCC after SVR. Older age, male sex, and progressive liver fibrosis have been reported as the risk factors [11-17]. Several reports, including ours, suggested that obesity was associated with the development of HCC in chronic viral hepatitis [18, 19]. However, it is still unclear whether obesity is also associated with the development of HCC after SVR.

Although a consensus has been reached regarding the fact that achieving SVR with IFN-based therapy reduces the risk of hepatocarcinogenesis $[12,20,21]$, controversies exist with regard to whether DAA therapy increases or decreases the risk of HCC occurrence or recurrence $[15,16,22,23]$. Recently, a large-scale observational study demonstrated that DAA therapy was associated with reduced risk for mortality and development of HCC [17]. However, in this single-arm study, the patients started DAA therapy after enrollment. The time-dependent Cox regression and pseudo Kaplan-Meier methods were used to show the benefit of DAA therapy. Therefore, the verification of these results in another cohort with controls could clarify this evidence.

In this study, we aimed to evaluate the rates of HCC development after achievement of SVR with antiviral therapies in patients with chronic HCV infection without prior history of HCC, to investigate the risk factors associated with the development of HCC including lifestyle factors such as obesity, diabetes mellitus, or alcohol consumption on hepatocarcinogenesis after SVR, and to compare between patients who achieved SVR with IFNbased and DAA therapy.

\section{Patients and Methods}

Study Design and Participants

In this multicenter retrospective cohort study, we enrolled patients aged over 18 years who were treated for chronic HCV infection with antiviral therapy and achieved SVR from April 1990 to December 2018. SVR was defined as undetectable HCV RNA at 24 weeks after the cessation of antiviral therapy. The exclusion criteria were as follows: (1) positive serology for hepatitis B surface antigen or coinfection with human immunodeficiency virus, (2) prior history of HCC before achievement of SVR, (3) missing values of liver transaminase or platelet count at achievement of SVR, and (4) not being under regular surveillance. The enrolled patients were divided into 2 groups according to their antiviral treatment regimens, namely, the IFN group and the DAA group. Patients who achieved SVR through a combination of pegylated IFN, ribavirin (RBV), and single DAA agent were categorized as the IFN group. Patient profiles and hematological and biochemical data were collected at the achievement of SVR. Obesity was defined as a BMI of $25 \mathrm{~kg} / \mathrm{m}^{2}$ or more, according to the criteria of the Japan Society for 
Table 1. Baseline patient characteristics

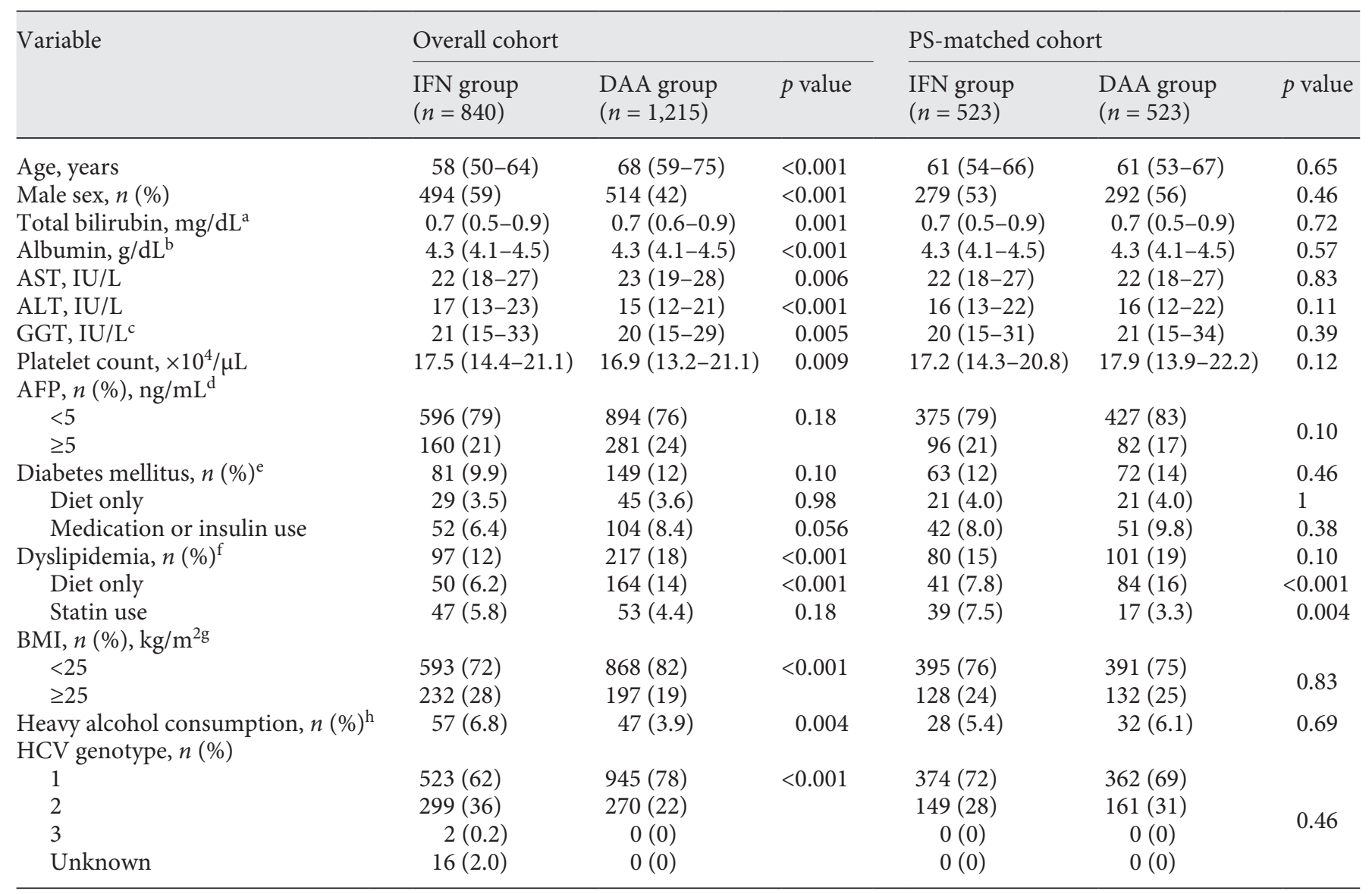

Values are medians (interquartile ranges) or numbers (\%). All values are of 24 weeks after cessation of antiviral therapies. The MannWhitney $\mathrm{U}$ test for continuous variables and the $\chi^{2}$ test for categorical variables were used to compare between the IFN and DAA groups. Data were missing for ${ }^{\mathrm{a}} 25,{ }^{\mathrm{b}} 23,{ }^{\mathrm{c}} 4,{ }^{\mathrm{d}} 124,{ }^{\mathrm{e}} 31,{ }^{\mathrm{f}} 29,{ }^{\mathrm{g}} 165$, and ${ }^{\mathrm{h}} 42$ participants. AFP, alpha-fetoprotein; ALT, alanine aminotransferase; AST, aspartate aminotransferase; DAA, direct-acting antivirals; GGT, $\gamma$-glutamyltransferase; HCV, hepatitis C virus; IFN, interferon; PS, propensity score.

the Study of Obesity [24], and heavy alcohol consumption was defined as a daily alcohol consumption of $60 \mathrm{~g}$ or more. Diabetes mellitus was defined as hemoglobin A1c level $\geq 6.5 \%$ following the Japanese clinical guideline [25], use of diabetes medications or insulin therapy, or presence of a medical diagnosis by an attending physician in the medical record. Dyslipidemia was defined as lowdensity lipoprotein cholesterol $\geq 140 \mathrm{mg} / \mathrm{dL}$, triglycerides $\geq 150$ $\mathrm{mg} / \mathrm{dL}$, or high-density lipoprotein cholesterol $<40 \mathrm{mg} / \mathrm{dL}$ following the Japanese clinical guideline [26]; or total cholesterol $\geq 220$ $\mathrm{mg} / \mathrm{dL}$ following the previous diagnostic criteria; use of medications for dyslipidemia; or presence of a medical diagnosis by an attending physician in the medical record.

\section{Antiviral Therapy}

The IFN therapy regimens were as follows: IFN monotherapy for 24-48 weeks for genotypes 1 and 2; IFN plus RBV for 48-72 weeks for genotype 1 and 24 weeks for genotype 2; peginterferon (PEG-IFN) monotherapy for 24-48 weeks for genotypes 1 and 2;
PEG-IFN plus RBV for 48-72 weeks for genotype 1 and 24 weeks for genotype 2 or 3; and PEG-IFN plus RBV plus telaprevir, simeprevir, or vaniprevir for 12 weeks followed by 12 weeks of PEG-IFN plus RBV for genotype 1 . The DAA therapy regimens were as follows: daclatasvir plus asunaprevir for 24 weeks for genotype 1; sofosbuvir plus ledipasvir for 12 weeks for genotype 1; sofosbuvir plus RBV for 12 weeks for genotype 2; ombitasvir and paritaprevir with ritonavir for 12 weeks for genotype 1; elbasvir plus grazoprevir for 12 weeks for genotype 1; daclatasvir plus asunaprevir plus beclabuvir for 12 weeks for genotype 1 ; and glecaprevir plus pibrentasvir for $8-12$ weeks for genotypes 1 and 2 .

\section{Surveillance for HCC and Diagnosis of HCC}

The surveillance protocol was in accordance with the Japanese Clinical Practice Guidelines: abdominal ultrasonography every 3-6 months with determination of tumor markers and optional CT or MRI every 6-12 months in patients whose ultrasonography images had poor visualization [27]. If HCC was suspected on the basis of 
Fig. 1. Cumulative probability and KaplanMeier estimates of development of HCC. a Cumulative probability of development of HCC by treatment groups. Incidence rates of $\mathrm{HCC}$ at 1,2 , and 3 years were 0.7 , 0.7 , and $2.0 \%$, respectively, in the IFN group and $1.4,2.8$, and $3.7 \%$, respectively, in the DAA group. $\mathbf{b}$ Cumulative probability of development of HCC according to BMI. Incidence rates of $\mathrm{HCC}$ at 1, 2, and 3 years were $2.2,3.5$, and $6.8 \%$, respectively, in patients with obesity (BMI $\geq 25 \mathrm{~kg} / \mathrm{m}^{2}$ ) and $0.9,1.6$, and $2.0 \%$, respectively, in patients without obesity. c Cumulative probability of development of HCC according to alcohol consumption. Incidence rates of HCC at 1, 2, and 3 years were 2.0, 2.0, and $9.1 \%$, respectively, in patients with heavy alcohol consumption ( $\geq 60 \mathrm{~g} /$ day) and 1.1, 1.9 , and $2.7 \%$, respectively, in patients without heavy alcohol consumption. DAA, direct-acting antiviral; HCC, hepatocellular carcinoma; IFN, interferon; SVR, sustained virological response.

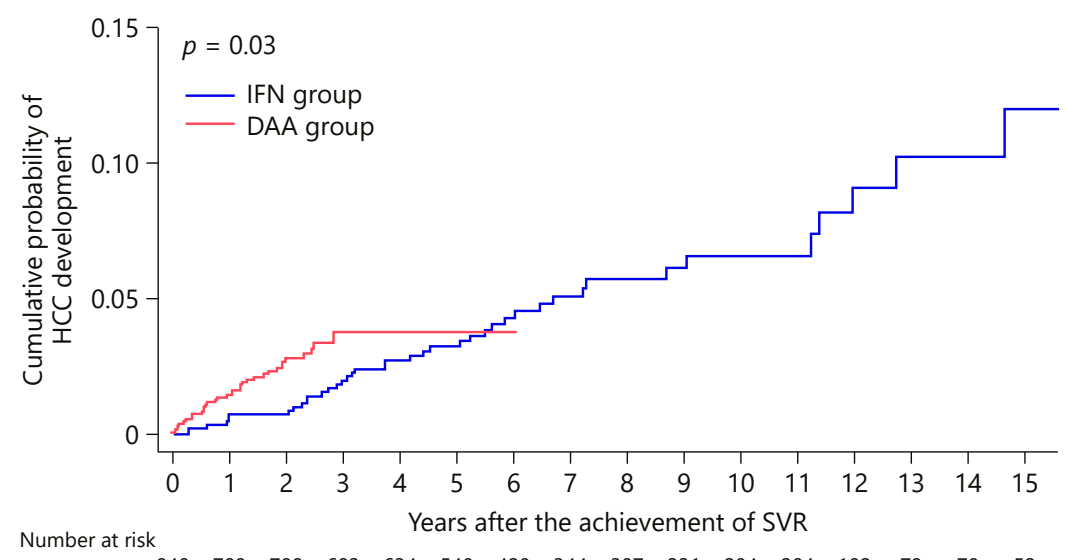

Number at risk

Years after the achievement of SVR

a $\quad \begin{array}{rrrr}1,215 & 1,089 \quad 803 \quad 242\end{array}$

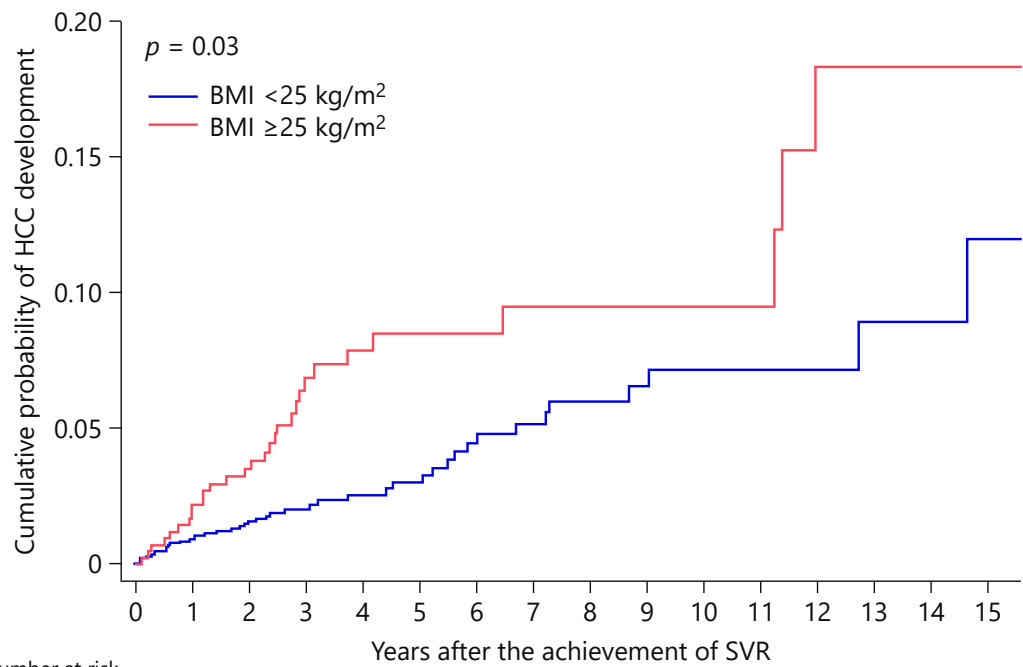

Number at risk

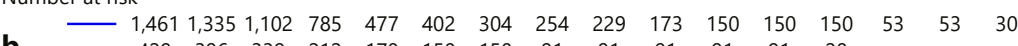

$\begin{array}{llllllllllllll}4 & 429 & 396 & 339 & 212 & 170 & 150 & 150 & 91 & 91 & 91 & 91 & 91 & 28\end{array}$

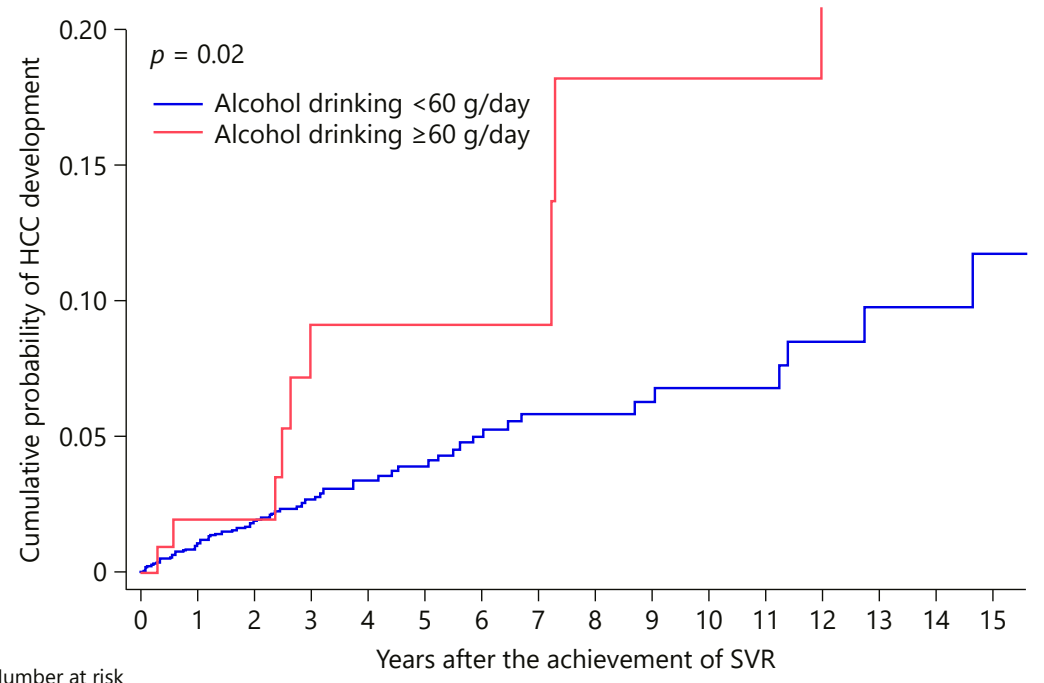

Number at risk

Years after the achievement of SVR

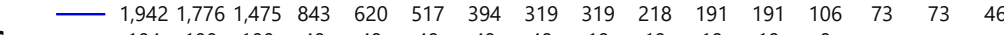

$\begin{array}{llllllllllllll} & 104 & 100 & 100 & 48 & 48 & 48 & 48 & 48 & 19 & 19 & 19 & 19 & 8\end{array}$ 
Fig. 2. Cumulative probability and KaplanMeier estimates of development of HCC in propensity score-matched cohorts. There was no significant difference between the matched groups $(p=0.97$ by the log-rank test). DAA, direct-acting antiviral; HCC, hepatocellular carcinoma; IFN, interferon; SVR, sustained virological response.

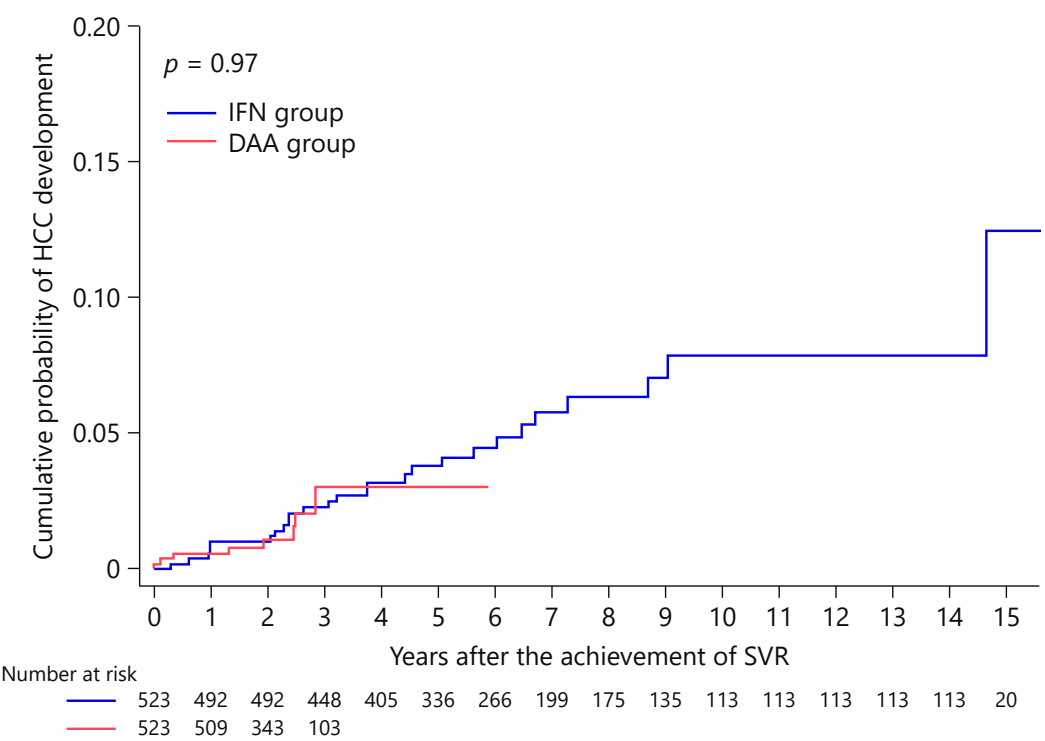

ultrasonographic results, additional procedures, such as dynamic CT or MRI, were used to confirm the diagnosis. Imaging findings leading to a definitive diagnosis of HCC were hyperattenuation in the arterial phase and washout in the late phase in dynamic CT or MRI $[28,29]$. We also collected survival status of the patients. The cause of death was determined by the attending physician.

\section{Statistical Analysis}

Data are presented as medians and interquartile ranges for quantitative variables and as numbers and percentages for qualitative variables. Differences between the IFN and DAA groups were evaluated using the Mann-Whitney $U$ test for continuous variables and the $\chi^{2}$ test for categorical variables. To assess the relevant trends of HCC characteristics identified after achievement of SVR, the Cochran-Armitage trend test was used.

Time to incidence of HCC was defined as the interval between the achievement of SVR and the definitive diagnosis of HCC. HCC incidence rates from the achievement of SVR were assessed using the Kaplan-Meier method and compared using the log-rank test. Patients who were lost to follow-up or those who died without HCC were censored. Follow-up was censored on June 30, 2019. Factors such as age, sex, total bilirubin, albumin, aspartate aminotransferase, alanineaminotransferase(ALT), $\gamma$-glutamyltransferase (GGT), platelet count, alpha-fetoprotein (AFP), presence or absence of diabetes mellitus and dyslipidemia, BMI, heavy alcohol consumption, and antiviral regimen were analyzed as risk factors for development of HCC by univariate and multivariate Cox proportional hazard regression analyses. Multivariate analysis was conducted by entering all variables with a $p<0.10$ in the univariate analysis. We also plotted the logarithmic hazard ratios for BMI and alcohol consumption using the restricted cubic spline method. In addition, a propensity score matching analysis was performed to compare HCC occurrence rates. Propensity scores were calculated by logistic regression based on age, sex, platelet count, HCV genotype, total bilirubin, obesity, and dyslipidemia. Nearest-neighbor matching at a ratio of $1: 1$ was applied with a caliper of 0.01 . All tests for differences were two-tailed, and a $p$ value $<0.05$ was considered to indicate a statistically significant difference. Statistical analyses were performed using $\mathrm{R}$ software (version 3.6.1; R Development Core Team, Vienna, Austria).

\section{Results}

\section{Patient Profiles}

A total of 2,055 patients were included in this study and divided into the IFN group $(n=840)$ and the DAA group $(n=1,215)$. The baseline characteristics of the patients in the 2 groups at the achievement of SVR are shown in Table 1. Patients in the DAA group were significantly older than those in the IFN group (median age, 68 vs. 58 years, $p<0.001$ ). The proportion of female patients was higher in the DAA group than in the IFN group (58 and 41\%, respectively; $p<0.001$ ). Compared with patients in the IFN group, those in the DAA group had lower ALT levels, GGT levels, platelet counts, and BMI. In the DAA group, 263 patients $(22 \%)$ had a history of treatment with IFN-based therapy.

\section{HCC Development}

Among 2,055 patients in the entire cohort, 75 patients (41 in the IFN group and 34 in the DAA group) developed HCC during the mean observation period of 4.1 (6.8 in the IFN group and 2.2 in the DAA group) years. The incidence rates of $\mathrm{HCC}$ at 1,2 , and 3 years were $1.2,1.9$, and $3.0 \%$, respectively, in the entire cohort; $0.7,0.7$, and $2.0 \%$, 
Table 2. Univariate and multivariate analyses of development of HCC in the entire cohort

\begin{tabular}{|c|c|c|c|c|}
\hline \multirow[t]{2}{*}{ Variable } & \multicolumn{2}{|l|}{ Univariate } & \multicolumn{2}{|l|}{ Multivariate } \\
\hline & $\operatorname{HR}(95 \% \mathrm{CI})$ & $p$ value & HR (95\% CI) & $p$ value \\
\hline Age per 1 year & $1.07(1.04-1.10)$ & $<0.001$ & $1.09(1.05-1.12)$ & $<0.001$ \\
\hline Male sex & $1.49(0.93-2.37)$ & 0.09 & $1.43(0.84-2.45)$ & 0.19 \\
\hline Total bilirubin per $1.0 \mathrm{mg} / \mathrm{dL}$ & $2.02(1.38-2.97)$ & $<0.001$ & $1.21(0.71-2.05)$ & 0.48 \\
\hline Albumin per $1.0 \mathrm{~g} / \mathrm{dL}$ & $0.36(0.18-0.57)$ & $<0.001$ & $0.48(0.25-0.94)$ & 0.03 \\
\hline AST per $10 \mathrm{IU} / \mathrm{L}$ & $1.24(1.11-1.38)$ & $<0.001$ & $0.93(0.72-1.19)$ & 0.55 \\
\hline ALT per $10 \mathrm{IU} / \mathrm{L}$ & $1.09(1.00-1.19)$ & 0.06 & $0.98(0.79-1.22)$ & 0.87 \\
\hline GGT per $10 \mathrm{IU} / \mathrm{L}$ & $1.04(1.01-1.07)$ & 0.004 & $1.03(0.97-1.10)$ & 0.29 \\
\hline Platelet count per $1 \times 10^{4} / \mu \mathrm{L}$ & $0.86(0.82-0.91)$ & $<0.001$ & $0.93(0.88-0.98)$ & 0.005 \\
\hline $\mathrm{AFP} \geq 5 \mathrm{ng} / \mathrm{mL}$ & $3.45(2.17-5.48)$ & $<0.001$ & $2.77(1.66-4.64)$ & $<0.001$ \\
\hline Diabetes & $1.70(0.91-3.15)$ & 0.09 & $1.89(0.98-3.66)$ & 0.057 \\
\hline Diet only & $1.97(0.79-4.91)$ & 0.14 & & \\
\hline Medication or insulin use & $1.54(0.70-3.37)$ & 0.28 & & \\
\hline Dyslipidemia & $0.16(0.04-0.65)$ & 0.01 & $0.16(0.04-0.66)$ & 0.01 \\
\hline Diet only & $\mathrm{na}^{\mathrm{a}}$ & na & & \\
\hline Statin use & $0.39(0.10-1.59)$ & 0.19 & & \\
\hline $\mathrm{BMI} \geq 25 \mathrm{~kg} / \mathrm{m}^{2}$ & $2.09(1.32-3.34)$ & 0.002 & $2.53(1.51-4.25)$ & $<0.001$ \\
\hline Heavy alcohol consumption & $2.24(1.12-4.51)$ & 0.02 & $2.56(1.14-5.75)$ & 0.02 \\
\hline Genotype 1 & $1.41(0.83-2.38)$ & 0.20 & & \\
\hline DAA versus IFN & $1.90(1.06-3.39)$ & 0.03 & $1.19(0.61-2.33)$ & 0.61 \\
\hline
\end{tabular}

Independent risk factors for HCC development were analyzed using Cox proportional hazard regression analyses. AFP, alpha-fetoprotein; ALT, alanine aminotransferase; AST, aspartate aminotransferase; CI, confidence interval; DAA, direct-acting antivirals; GGT, $\gamma$-glutamyltransferase; HCC, hepatocellular carcinoma; HR, hazard ratio; IFN, interferon. ${ }^{\text {a }}$ Hazard ratio for dyslipidemia with only diet could not be calculated because there was no case of HCC development in this category.

respectively, in the IFN group; and 1.4, 2.8, and 3.7\%, respectively, in the DAA group (Fig. 1a). There was a significant difference in the incidence rates of HCC between the 2 groups ( $p=0.03$ by the log-rank test).

Propensity score matching analysis was performed, and 523 patients in each group were extracted. The baseline characteristics of matched groups are shown in Table 1. Factors other than statin use and dietary for dyslipidemia were well matched. There was no significant difference in incidence rates of HCC between the matched groups ( $p=0.97$ by the log-rank test) (Fig. 2).

In the subgroup analysis, lifestyle factors - obesity $\left(\mathrm{BMI} \geq 25 \mathrm{~kg} / \mathrm{m}^{2}\right)$ and heavy alcohol consumption ( $\geq 60 \mathrm{~g} /$ day) - were significant risk factors for development of HCC. The cumulative incidence rates of HCC at 1,2, and 3 years were $2.2,3.5$, and $6.8 \%$, respectively, in patients with obesity and $0.9,1.6$, and $2.0 \%$, respectively, in those without obesity ( $p=0.001$, Fig. $1 \mathrm{~b})$. The incidence rates at 1,2 , and 3 years were $2.0,2.0$, and $9.1 \%$, respectively, in patients with heavy alcohol consumption and 1.1, 1.9, and $2.7 \%$, respectively, in those without heavy alcohol consumption (Fig. 1c, $p=0.02$ ).

\section{Risk Factors for Development of HCC}

In univariate analyses, the following factors were associated with an increased risk of development of HCC $(p<0.10)$ : older age, male sex, higher total bilirubin level, lower albumin level, higher aspartate aminotransferase level, higher ALT level, higher GGT level, lower platelet count, higher AFP level, diabetes mellitus, absence of dyslipidemia, higher BMI, heavy alcohol consumption, and antiviral therapy with DAA. Multivariate analysis, adjusted for the factors with a $p<0.10$ in univariate analyses, identified older age, lower albumin level, lower platelet count, higher AFP level, absence of dyslipidemia, higher $\mathrm{BMI}$, and heavy alcohol consumption as independent risk factors for development of HCC (Table 2). There was no significant difference in the development of HCC between the IFN group and the DAA group (HR, 1.21; $p=$ 0.58 ). The dose-dependent risk curves of BMI and alcohol consumption are shown in Figures $3 a$ and $b$.

\section{Characteristics of HCC}

The characteristics of HCC are shown in Table 3. Most patients were diagnosed at an early stage; $90 \%$ had a single 
tumor, and the tumor size was $3 \mathrm{~cm}$ or smaller in $93 \%$ of the patients. There was no significant difference in terms of the characteristics of HCC between the 2 groups. As a result, $>90 \%$ of patients from both the groups underwent surgical resection or ablation as an initial treatment.

\section{Survival and Cause of Death}

Twenty-one patients had died before HCC diagnosis during the observation period. Extrahepatic malignancies were the leading cause of death, including 4 cases of lung cancer, 2 cases each of pancreatic cancer and cervical cancer, and 1 case each of stomach cancer, rectal cancer, and malignant lymphoma. Cerebrovascular disease was responsible for 2 patients and pneumonia for 1 . The cause of the other 7 deaths was unknown. No deaths due to liver failure were observed.

\section{Discussion}

In this large multicenter cohort study, we identified 75 cases of HCC and demonstrated that obesity and heavy alcohol consumption were risk factors for HCC in patients with chronic hepatitis $\mathrm{C}$ who achieved SVR. Meanwhile, we did not observe an increased risk of HCC in patients treated with DAA rather than IFN therapy.

Obesity was previously reported to be a risk factor for various cancers, including HCC, in a large-scale epidemiological research [30], and it exerts synergistic effects on hepatocarcinogenesis in patients with active viral infection $[18,19]$. Several mechanisms of hepatocarcinogenesis in patients with obesity have been proposed, as follows: increased levels of inflammatory cytokines such as tumor necrosis factor- $\alpha$ or interluekin-6 [31], hyperinsulinemia caused by insulin resistance [32], adipokines secreted in the adipose tissue [33], oxidative stress and lipotoxicity [34], changes in intestinal flora [35], and progression to nonalcoholic steatohepatitis [36-38]. Through these multiple pathways, obesity might affect hepatocarcinogenesis even after achievement of SVR.

It has been well documented that excess alcohol consumption is one of the major causes of HCC [39]. Previous reports advocated that alcohol consumption had a synergistic effect on the development of HCC in patients with chronic viral hepatitis $[39,40]$ and increased the risk of HCC in patients after SVR [13,41]. In the current study, heavy alcohol consumption, defined as consumption of $60 \mathrm{~g}$ or more alcohol per day, was associated with an increased risk of developing HCC. Dose-dependent risk curve of HCC development for the amount of alco-

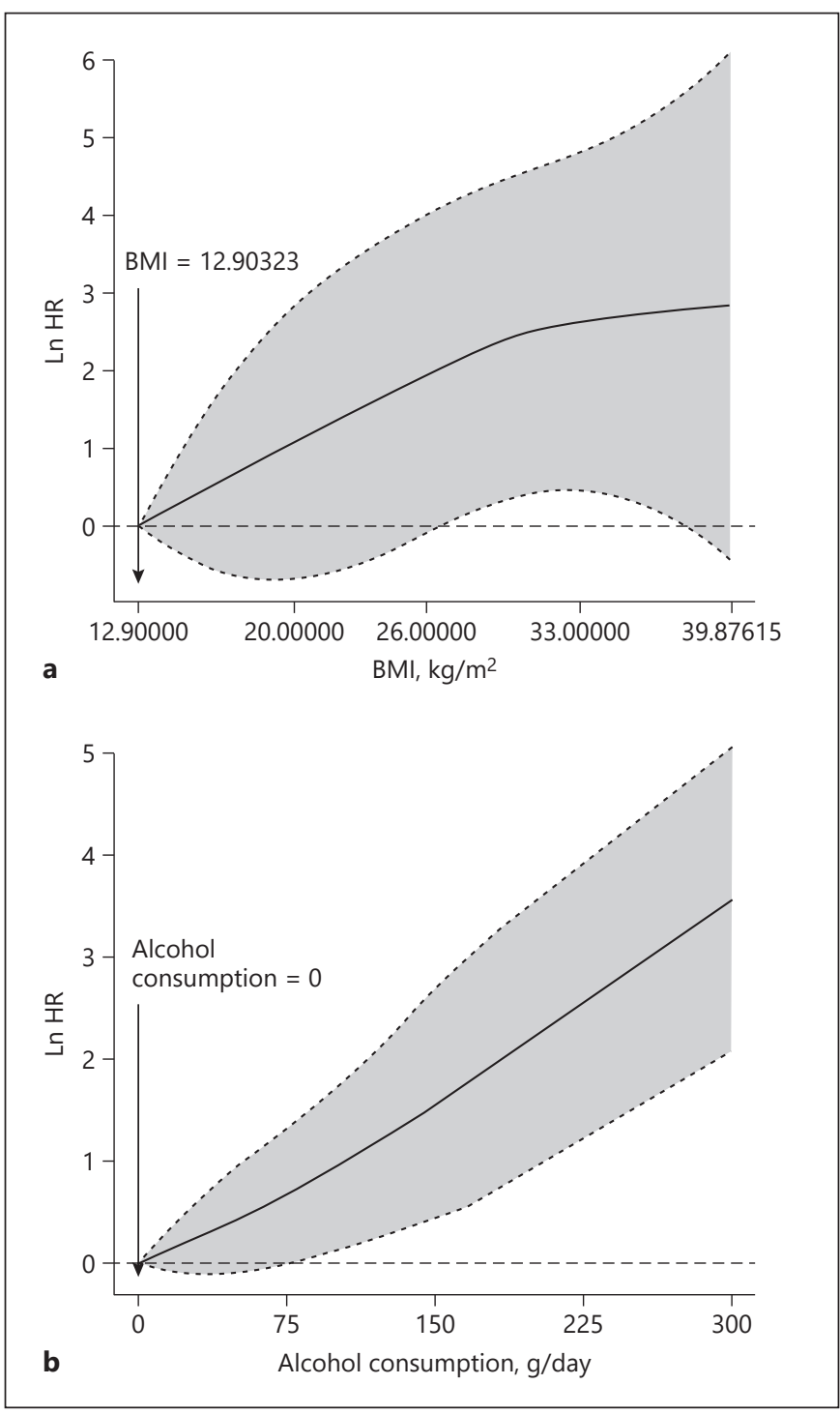

Fig. 3. Smooth log hazard ratio of HCC development. a Smooth log hazard ratio for BMI. The solid line represents the estimated log hazard ratio of HCC development for BMI adjusted for age, albumin level, platelet count, AFP level, and presence of dyslipidemia and heavy alcohol consumption, and the dotted lines represent the 95\% confidence interval. b Smooth log hazard ratio for the amount of alcohol consumption. The solid line represents the estimated log hazard ratio of HCC development for the amount of alcohol consumption adjusted for age, albumin level, platelet count, AFP level, and presence of dyslipidemia and obesity, and the dotted lines represent the $95 \%$ confidence interval. HR, hazard ratio; HCC, hepatocellular carcinoma; AFP, alpha-fetoprotein.

hol consumption represented that the less the alcohol intake, the lower the risk of HCC (Fig. 3b). The result suggested that even moderate drinking can be a risk for HCC. 
Table 3. Characteristics of HCC identified after achievement of SVR

\begin{tabular}{|c|c|c|c|}
\hline Characteristic & $\begin{array}{l}\text { IFN group } \\
(n=41)\end{array}$ & $\begin{array}{l}\text { DAA group } \\
(n=34)\end{array}$ & $p$ value \\
\hline \multicolumn{4}{|l|}{ Largest tumor size, $\mathrm{cm}$} \\
\hline$\leq 2$ & $23(56)$ & $27(79)$ & \multirow{3}{*}{0.32} \\
\hline $2.1-3$ & $17(41)$ & $3(8.8)$ & \\
\hline$>3$ & $1(2.4)$ & $4(12)$ & \\
\hline \multicolumn{4}{|l|}{ Intrahepatic tumor number } \\
\hline Single & $36(88)$ & $31(91)$ & \multirow{3}{*}{0.96} \\
\hline $2-3$ & $5(12)$ & $2(5.9)$ & \\
\hline$>3$ & $0(0)$ & $1(2.9)$ & \\
\hline Portal vein invasion, $n(\%)$ & $2(4.9)$ & $1(2.9)$ & 0.87 \\
\hline Extrahepatic metastasis, $n(\%)$ & $0(0)$ & $2(5.9)$ & 0.39 \\
\hline \multicolumn{4}{|l|}{ Surveillance interval, months } \\
\hline$\leq 6$ & $32(78)$ & $31(91)$ & \multirow{2}{*}{0.22} \\
\hline$>6$ & $9(22)$ & $3(8.8)$ & \\
\hline \multicolumn{4}{|l|}{ Treatment for HCC } \\
\hline Surgical resection & $15(37)$ & $4(12)$ & \multirow{5}{*}{0.11} \\
\hline Ablation & $23(56)$ & $27(79)$ & \\
\hline TACE combined with radiation & $1(2.4)$ & $1(2.9)$ & \\
\hline Systemic chemotherapy & $0(0)$ & $1(2.9)$ & \\
\hline na & $2(4.9)$ & $1(2.9)$ & \\
\hline
\end{tabular}

Values are numbers (\%). The Cochran-Armitage trend test for tumor size and number and the $\chi^{2}$ test for other categorical variables were used to compare them between the IFN and DAA groups. DAA, direct-acting antivirals; HCC, hepatocellular carcinoma; IFN, interferon; na, not available; TACE, transcatheter arterial chemoembolization; SVR, sustained virological response.

However, conventional risk factors for HCC such as age, male sex, liver fibrosis progression, and higher AFP level are unmodifiable, and physicians can intervene in lifestyle factors such as obesity and heavy drinking. Further investigation is warranted to elucidate whether lifestyle modifications such as weight reduction or moderation in alcohol consumption after achievement of SVR can reduce the risk of disease progression and, eventually, development of HCC. However, it is highly reasonable to recommend patients to refrain from being overweight and from consuming alcohol.

Diabetes mellitus was previously reported to be a significant risk factor for HCC development [42]. In this study, diabetes was a risk factor with marginal significance $(p=0.057)$ in multivariate analysis. Obesity and diabetes are both components of metabolic syndrome, thus sharing insulin resistance as a background condition. Insulin resistance leading to increased levels of insulin and insulin-like growth factors is supposed to play a major role in hepatocarcinogenesis in obesity-associated HCC [43]. It is still controversial whether insulin therapy or insulin-secreting agents increase the risk of cancer development. A metaanalysis of 5 case-control studies and 10 cohort studies re- vealed that insulin treatment was associated with an increased risk of overall cancer but not with HCC [44]. Sulfonylureas treatment was reported to be associated with an increased risk of cancer compared with metformin $[45,46]$. In contrast, a meta-analysis of randomized control studies revealed that dipeptidyl peptidase- 4 inhibitors were not associated with short-term increased cancer risk [47]. In this study, detailed data on the medication were not available, and it was unclear whether a specific medication affected HCC incidence. We observed an inverse association between dyslipidemia and HCC incidence in this study. There was a similar report of the inverse association from a prospective study of 578,700 subjects in Norway, Austria, and Sweden [48]. The mechanism was uncertain; however, it might be due to reduced cholesterol synthesis in cirrhosis. We performed an ad hoc analysis to compare platelet count between those with and without dyslipidemia. As a result, the platelet count was higher in those with dyslipidemia (data not shown). There were several reports regarding statin use and reduced HCC risk. One report documented universal risk reduction [49], and another report documented that only lipophilic statins, not hydrophilic statins, were associated with reduced HCC incidence [50]. The ef- 
fect of statin use on HCC development was not conclusive in this study partly because the number of statin users was small, and the type of statin was not available.

There has been an argument that DAA can reduce the risk of HCC to an extent equal to that shown by IFNbased therapy. In this study, the crude rates of development of HCC were higher in the DAA group than in the IFN group. As some patients in the DAA group were ineligible for or nonresponders to IFN-based therapy, the median patient age was higher and platelet count was lower in the DAA group than in the IFN group. Therefore, after adjustment by baseline factors, there was no significant difference in the risk for development of HCC between the 2 groups. The propensity score matching analysis further confirmed the results.

To the best of our knowledge, this study had the longest follow-up period of up to 15 years after achievement of SVR among similar studies published so far. The exact duration for which patients should be followed up to evaluate HCC development after SVR is still unclear. In this study, the cumulative incidence of HCC estimated by the Kaplan-Meier method never plateaued over 10 years. It should be noted that a longer, probably lifelong, followup period is necessary for high-risk patients [12, 51, 52].

This study has several limitations. First, the observation period of the patients in the DAA group was relatively short because DAA treatments have been recently introduced. Second, we adopted $25 \mathrm{~kg} / \mathrm{m}^{2}$ as the cutoff value of obesity according to the criteria of the Japan Society for the Study of Obesity because few patients in our cohort had a BMI $\geq 30 \mathrm{~kg} / \mathrm{m}^{2}$, which corresponds to the definition of obesity according to the World Health Organization criteria [53]. In addition, owing to a higher prevalence of type 2 diabetes and cardiovascular disease among Asian individuals than among Caucasian individuals with the same BMI, lowering of BMI cutoff points for obesity in Asian individuals has been explored [54, 55]. However, it is still uncertain whether the results could be applied to non-Asian individuals. Third, risk factors for HCC development were collected only at enrollment. Because subsequent data after SVR were not available, we could not evaluate the impact of lifestyle modification after SVR. Fourth, detailed data on duration of drinking and cumulative alcohol intake were not available.

In conclusion, obesity and heavy alcohol consumption were independent risk factors for development of HCC after achievement of SVR. Further studies are required to investigate whether lifestyle modification could reduce the risk of HCC. Our findings also suggested that along with IFN-based therapy, DAA therapy could also reduce the risk of development of HCC.

\section{Appendix}

SMART-C study group consisted of the following investigators: Shintaro Mikami, Mikio Yanase, and Naohiko Masaki (Center Hospital of the National Center for Global Health and Medicine, Tokyo); Toshiro Kamoshida (Hitachi General Hospital, Hitachi); Hiroyoshi Taniguchi and Hideo Yoshida (Japanese Red Cross Medical Center, Tokyo), Masatoshi Akamatsu (JR Tokyo General Hospital, Tokyo); Eriko Goto, Osamu Togawa, and Yukihiro Koike (Kanto Central Hospital of the Mutual Aid Association of Public School Teacher, Tokyo); Itsuro Ogata (Kawakita General Hospital, Tokyo); Norie, Yamada, and Kiyomi Yasuda (Kiyokawa Hospital, Tokyo); Kiyohiko Kurai (Kurai Kiyohiko Medical Clinic, Utsunomiya); Toshihiro Kawai, Takahisa Sato, Yuji Kondo, Shinpei Sato, and Shuntaro Obi (Kyoundo Hospital, Tokyo); Mayuko Kondo and Takamasa Oki (Mitsui Memorial Hospital, Tokyo); Takashi Ishikawa (Marunouchi Clinic, Tokyo); Kayo Nagashima and Hideo Kamiichi (National Disaster Medical Center, Tachikawa); Takuma Teratani (NTT Medical Center Tokyo, Tokyo); Kazuyuki Hanajiri and Tomonori Wada (Sanraku Hospital, Tokyo); Kazuaki Inoue (Showa University Fujigaoka Hospital, Yokohama); Yoriyuki Takamori, Yoshinari Asaoka, and Atsushi Tanaka (Teikyo University School of Medicine, Tokyo); Keiji Ogura (Tokyo Metropolitan Police Hospital, Tokyo); Hiroshi Mitsui (Tokyo Postal agency Hospital, Tokyo); Hajime Fujie (Tokyo Shinjuku Medical Center, Tokyo); Kenji Hirano and Hisato Maekawa (Tokyo Takanawa Hospital, Tokyo); Toru Arano, Kazuaki Tejima, and Masahiro Arai (Toshiba Hospital, Tokyo); Hiroshi Yotsuyanagi (University of Tokyo, the Institute of Medical Science, Tokyo); Tatsuya Minami, Ryosuke Tateishi, Naoto Fujiwara, Ryo Nakagomi, Takuma Nakatsuka, Masaya Sato, Kenichiro Enooku, Hayato Nakagawa, Kazuyuki Hanajiri, Masashi Izumiya, Hidetaka Fujinaga, Yoshinari Asaoka, Yuji Kondo, Yasuo Tanaka, Motoyuki Otsuka, Naoya Kato, Kyoji Moriya, and Kazuhiko Koike (University of Tokyo Graduate School of Medicine, Tokyo).

\section{Acknowledgement}

We would like to thank Editage (www.editage.com) for English language editing.

\section{Statement of Ethics}

This study complied with the guidelines for human studies and was conducted in accordance with the World Medical Association Declaration of Helsinki and with the ethical guidelines for epidemiological research of the Japanese Ministry of Education, Culture, Sports, Science, and Technology and the Ministry of Health, Labor, and Welfare. The cohort of patients was enrolled in the University of Tokyo Hospital and its affiliated hospitals participating in the Specific Molecule Antiviral tReatment Tokyo Hepatitis C (SMART-C) Group. The study protocol was approved by the University of Tokyo Medical Research Center Ethics Committee (Approval No. 10975) and the Institutional Review Board or Ethics 
Committee of each participating institution. Written informed consent was obtained from each patient, and opt-out was also permitted in case of loss to follow-up. This study was registered in the University Hospital Medical Information Network (UMIN) Clinical Trial Registry (UMIN ID: 000023997).

\section{Conflict of Interest Statement}

Kazuhiko Koike has received research funding from Merck Sharp \& Dohme, Chugai Pharmaceutical Co., Ltd, Bristol-Meyers Squibb, Gilead Sciences, AbbVie GK, Janssen Pharmaceutical K.K., and Mitsubishi Tanabe Pharma Corporation. Ryosuke Tateishi has received lecture fee from Merck Sharp \& Dohme, Chugai Pharmaceutical Co., Ltd, Bristol-Meyers Squibb, Gilead Sciences, AbbVie GK, and Mitsubishi Tanabe Pharma Corporation. Tatsuya Minami has received lecture fee from Merck Sharp \& Dohme, Gilead Sciences, and AbbVie GK. Hiroshi Yotsuyanagi has received lecture fee from Merck Sharp \& Dohme, Bristol-Meyers Squibb, Gilead Sciences, and AbbVie GK. Naoya Kato has received research funding and lecture fee from Merck Sharp \& Dohme, Chugai Pharmaceutical Co., Ltd, Bristol-Meyers Squibb, Gilead Sciences, AbbVie GK, Jansen Pharmaceutical K.K., and Mitsubishi Tanabe Pharma Corporation. Atsushi Tanaka has received lecture fee from Gilead Sciences and AbbVie GK. Yoshinari Asaoka has received lecture fee from Merck Sharp \& Dohme, Gilead Sciences, and AbbVie GK. Kazuaki Inoue has received research funding from Chugai Pharmaceutical K.K, Bristol-Myers Squibb, AbbVie GK, and Merck Sharp \& Dohme and has received lecture fee from AbbVie GK, Bristol-Myers Squibb, Merck Sharp \& Dohme, and Gilead Sciences. Yasuo Tanaka has received research funding from Gilead Sciences. Hayato Nakagawa has received lecture fee from Merck Sharp \& Dohme and Gilead Sciences. Takamasa Ohki has received lecture fee from Bayer Yakuhin Ltd and Eisai Co., Ltd.

\section{Funding Sources}

This research was supported by the Research Program on Hepatitis from the Japan Agency for Medical Research and Development, AMED, under Grant No. JP17fk0210106, JP18fk0210022, 19fk0210022h0803, and 20fk0210066h0701.

\section{Author Contributions}

Conception and design, analysis and interpretation of the data, and drafting of the manuscript: Tatsuya Minami and Ryosuke Tateishi. Statistical analysis: Tatsuya Minami, Naoto Fujiwara, and Ryosuke Tateishi. Study supervision: Ryosuke Tateishi and Kazuhiko Koike. Acquisition of the data, final approval, and agreement to be accountable for all aspects of the work: all of the authors.

\section{References}

1 Global prevalence and genotype distribution of hepatitis C virus infection in 2015: a modelling study. Lancet Gastroenterol Hepatol. 2017 Mar;2(3):161-76.

2 Simonetti RG, Cammà $\mathrm{C}$, Fiorello $\mathrm{F}$, Cottone M, Rapicetta M, Marino L, et al. Hepatitis C virus infection as a risk factor for hepatocellular carcinoma in patients with cirrhosis. A case-control study. Ann Intern Med. 1992 Jan 15;116(2):97-102.

3 De Mitri MS, Poussin K, Baccarini P, Pontisso P, D'Errico A, Simon N, et al. HCV-associated liver cancer without cirrhosis. Lancet. 1995 Feb 18;345(8947):413-5.

4 El-Serag HB. Epidemiology of viral hepatitis and hepatocellular carcinoma. Gastroenterology. 2012 May;142(6):1264-73.e1.

5 Afdhal N, Zeuzem S, Kwo P, Chojkier M, Gitlin N, Puoti M, et al. Ledipasvir and sofosbuvir for untreated HCV genotype 1 infection. N Engl J Med. 2014 May 15;370(20):1889-98.

6 Zeuzem S, Ghalib R, Reddy KR, Pockros PJ, Ben Ari Z, Zhao Y, et al. Grazoprevir-elbasvir combination therapy for treatment-naive cirrhotic and noncirrhotic patients with chronic hepatitis $C$ virus genotype 1,4 , or 6 infection: a randomized trial. Ann Intern Med. $2015 \mathrm{Jul}$ 7;163(1):1-13.

7 Manns M, Samuel D, Gane EJ, Mutimer D, McCaughan G, Buti M, et al. Ledipasvir and sofosbuvir plus ribavirin in patients with genotype 1 or 4 hepatitis $C$ virus infection and advanced liver disease: a multicentre, openlabel, randomised, phase 2 trial. Lancet Infect Dis. 2016 Jun;16(6):685-97.

8 Forns X, Lee SS, Valdes J, Lens S, Ghalib R, Aguilar $\mathrm{H}$, et al. Glecaprevir plus pibrentasvir for chronic hepatitis $\mathrm{C}$ virus genotype $1,2,4$, 5 , or 6 infection in adults with compensated cirrhosis (EXPEDITION-1): a single-arm, open-label, multicentre phase 3 trial. Lancet Infect Dis. 2017 Oct;17(10):1062-8.

9 EASL recommendations on treatment of hepatitis C 2018. J Hepatol. 2018 Aug;69(2):461511.

10 Ghany MG, Morgan TR. Hepatitis C guidance 2019 update: American Association for the Study of Liver Diseases-Infectious Diseases Society of America Recommendations for Testing, Managing, and treating Hepatitis C Virus Infection. Hepatology. 2020 Feb;71(2): 686-721.

11 Yoshida H, Tateishi R, Arakawa Y, Sata M, Fujiyama S, Nishiguchi S, et al. Benefit of interferon therapy in hepatocellular carcinoma prevention for individual patients with chronic hepatitis C. Gut. 2004 Mar;53(3):425-30.

12 Asahina Y, Tsuchiya K, Nishimura T, MuraokaM,SuzukiY, TamakiN, etal. $\alpha$-Fetoprotein levels after interferon therapy and risk of hepatocarcinogenesis in chronic hepatitis C. Hepatology. 2013 Oct;58(4):1253-62.

13 Kanwal F, Kramer J, Asch SM, Chayanupatkul M, Cao Y, El-Serag HB. Risk of hepatocel- lular cancer in HCV patients treated with direct-acting antiviral agents. Gastroenterology. 2017 Oct;153(4):996-1005.e1.

14 Innes H, Barclay ST, Hayes PC, Fraser A, Dillon JF, Stanley A, et al. The risk of hepatocellular carcinoma in cirrhotic patients with hepatitis $\mathrm{C}$ and sustained viral response: role of the treatment regimen. J Hepatol. 2018 Apr;68(4):646-54.

15 Li DK, Ren Y, Fierer DS, Rutledge S, Shaikh OS, Lo Re V 3rd, et al. The short-term incidence of hepatocellular carcinoma is not increased after hepatitis $\mathrm{C}$ treatment with direct-acting antivirals: an ERCHIVES study. Hepatology. 2018 Jun;67(6):2244-53.

16 Nahon P, Layese R, Bourcier V, Cagnot C, Marcellin P, Guyader D, et al. Incidence of hepatocellular carcinoma after direct antiviral therapy for HCV in patients with cirrhosis included in surveillance programs. Gastroenterology. 2018 Nov; 155(5):1436-50.e6.

17 Carrat F, Fontaine H, Dorival C, Simony M, Diallo A, Hezode C, et al. Clinical outcomes in patients with chronic hepatitis $\mathrm{C}$ after direct-acting antiviral treatment: a prospective cohort study. Lancet. 2019 Apr 6;393(10179): 1453-64.

18 Chen CL, Yang HI, Yang WS, Liu CJ, Chen PJ, You SL, et al. Metabolic factors and risk of hepatocellular carcinoma by chronic hepatitis B/C infection: a follow-up study in Taiwan. Gastroenterology. 2008 Jul;135(1):111-21. 
19 Ohki T, Tateishi R, Sato T, Masuzaki R, Imamura J, Goto T, et al. Obesity is an independent risk factor for hepatocellular carcinoma development in chronic hepatitis $\mathrm{C} \mathrm{pa-}$ tients. Clin Gastroenterol Hepatol. 2008 Apr; 6(4):459-64.

20 Yoshida H, Shiratori Y, Moriyama M, Arakawa $\mathrm{Y}$, Ide $\mathrm{T}$, Sata $\mathrm{M}$, et al. Interferon therapy reduces the risk for hepatocellular carcinoma: national surveillance program of cirrhotic and noncirrhotic patients with chronic hepatitis $\mathrm{C}$ in Japan. IHIT Study Group. Inhibition of Hepatocarcinogenesis by Interferon Therapy. Ann Intern Med. 1999 Aug 3;131(3):174-81.

21 van der Meer AJ, Veldt BJ, Feld JJ, Wedemeyer H, Dufour JF, Lammert F, et al. Association between sustained virological response and all-cause mortality among patients with chronic hepatitis $\mathrm{C}$ and advanced hepatic fibrosis. JAMA. 2012 Dec 26;308(24):2584-93.

22 Conti F, Buonfiglioli F, Scuteri A, Crespi C, Bolondi L, Caraceni P, et al. Early occurrence and recurrence of hepatocellular carcinoma in HCV-related cirrhosis treated with directacting antivirals. J Hepatol. 2016 Oct;65(4): 727-33.

23 Reig M, Mariño Z, Perelló C, Iñarrairaegui M, Ribeiro A, Lens S, et al. Unexpected high rate of early tumor recurrence in patients with HCV-related HCC undergoing interferon-free therapy. J Hepatol. 2016 Oct;65(4):719-26.

24 New criteria for "obesity disease" in Japan. Circ J. 2002 Nov;66(11):987-92.

25 Seino Y, Seino Y, Nanjo K, Tajima N, Kadowaki T, Kashiwagi A, et al. Report of the committee on the classification and diagnostic criteria of diabetes mellitus. J Diabetes Investig. 2010 Oct 19;1(5):212-28.

26 Teramoto T, Sasaki J, Ishibashi S, Birou S, Daida H, Dohi S, et al. Executive summary of the Japan Atherosclerosis Society (JAS) guidelines for the diagnosis and prevention of atherosclerotic cardiovascular diseases in Japan-2012 version. J Atheroscler Thromb. 2013;20(6):517-23.

27 Kokudo N, Hasegawa K, Akahane M, Igaki H, Izumi N, Ichida T, et al. Evidence-based clinical practice guidelines for hepatocellular carcinoma: the Japan Society of Hepatology 2013 update (3rd JSH-HCC Guidelines). Hepatol Res. 2015 Jan;45(2):45.

28 Forner A, Vilana R, Ayuso C, Bianchi L, Solé $\mathrm{M}$, Ayuso JR, et al. Diagnosis of hepatic nodules $20 \mathrm{~mm}$ or smaller in cirrhosis: prospective validation of the noninvasive diagnostic criteria for hepatocellular carcinoma. Hepatology. 2008 Jan;47(1):97-104.

29 Sangiovanni A, Manini MA, Iavarone M, Romeo R, Forzenigo LV, Fraquelli M, et al. The diagnostic and economic impact of contrast imaging techniques in the diagnosis of small hepatocellular carcinoma in cirrhosis. Gut. 2010 May;59(5):638-44.
30 Bhaskaran K, Douglas I, Forbes H, dos-SantosSilva I, Leon DA, Smeeth L. Body-mass index and risk of 22 specific cancers: a populationbased cohort study of 5.24 million UK adults. Lancet. 2014 Aug 30;384(9945):755-65.

31 Park EJ, Lee JH, Yu GY, He G, Ali SR, Holzer $\mathrm{RG}$, et al. Dietary and genetic obesity promote liver inflammation and tumorigenesis by enhancing IL-6 and TNF expression. Cell. 2010 Jan 22;140(2):197-208.

32 Pollak M. Insulin and insulin-like growth factor signalling in neoplasia. Nat Rev Cancer. 2008 Dec;8(12):915-28.

33 Marra F, Bertolani C. Adipokines in liver diseases. Hepatology. 2009 Sep;50(3):957-69.

34 Neuschwander-Tetri BA. Hepatic lipotoxicity and the pathogenesis of nonalcoholic steatohepatitis: the central role of nontriglyceride fatty acid metabolites. Hepatology. 2010 Aug; 52(2):774-88

35 Yoshimoto S, Loo TM, Atarashi K, Kanda H, Sato S, Oyadomari S, et al. Obesity-induced gut microbial metabolite promotes liver cancer through senescence secretome. Nature. 2013 Jul 4;499(7456):97-101.

36 Bugianesi E, Leone N, Vanni E, Marchesini G, Brunello F, Carucci P, et al. Expanding the natural history of nonalcoholic steatohepatitis: from cryptogenic cirrhosis to hepatocellular carcinoma. Gastroenterology. 2002 Jul; 123(1):134-40.

37 Yasui K, Hashimoto E, Komorizono Y, Koike K, Arii S, Imai Y, et al. Characteristics of patients with nonalcoholic steatohepatitis who develop hepatocellular carcinoma. Clin Gastroenterol Hepatol. 2011 May;9(5):428-33; quiz e50.

38 Kanwal F, Kramer JR, Mapakshi S, Natarajan Y, Chayanupatkul M, Richardson PA, et al. Risk of hepatocellular cancer in patients with non-alcoholic fatty liver disease. Gastroenterology. 2018 Dec;155(6):1828-37.e2.

39 Morgan TR, Mandayam S, Jamal MM. Alcohol and hepatocellular carcinoma. Gastroenterology. 2004 Nov;127(5 Suppl 1):S87-96.

40 Vandenbulcke H, Moreno C, Colle I, Knebel JF, Francque S, Sersté T, et al. Alcohol intake increases the risk of HCC in hepatitis $\mathrm{C}$ virusrelated compensated cirrhosis: a prospective study. J Hepatol. 2016 Sep;65(3):543-51.

41 Yamashita N, Ohho A, Yamasaki A, Kurokawa M, Kotoh K, Kajiwara E. Hepatocarcinogenesis in chronic hepatitis $\mathrm{C}$ patients achieving a sustained virological response to interferon: significance of lifelong periodic cancer screening for improving outcomes. J Gastroenterol. 2014 Nov;49(11):1504-13.

42 El-Serag HB, Hampel H, Javadi F. The association between diabetes and hepatocellular carcinoma: a systematic review of epidemiologic evidence. Clin Gastroenterol Hepatol. 2006 Mar;4(3):369-80.
43 Karagozian R, Derdák Z, Baffy G. Obesity-associated mechanisms of hepatocarcinogenesis. Metab Clin Exp. 2014 May;63(5):607-17.

44 Janghorbani M, Dehghani M, Salehi-Marzijarani M. Systematic review and meta-analysis of insulin therapy and risk of cancer. Horm Cancer. 2012 Aug;3(4):137-46.

45 Currie CJ, Poole CD, Gale EA. The influence of glucose-lowering therapies on cancer risk in type 2 diabetes. Diabetologia. 2009 Sep; 52(9):1766-77.

46 Hsieh MC, Lee TC, Cheng SM, Tu ST, Yen $\mathrm{MH}$, Tseng $\mathrm{CH}$. The influence of type 2 diabetes and glucose-lowering therapies on cancer risk in the Taiwanese. Exp Diabetes Res. 2012;2012:413782.

47 Monami M, Dicembrini I, Martelli D, Mannucci E. Safety of dipeptidyl peptidase- 4 inhibitors: a meta-analysis of randomized clinical trials. Curr Med Res Opin. 2011 Nov; 27(Suppl 3):57-64.

48 Borena W, Strohmaier S, Lukanova A, Bjørge T, Lindkvist B, Hallmans G, et al. Metabolic risk factors and primary liver cancer in a prospective study of 578,700 adults. Int J Cancer. 2012 Jul 1;131(1):193-200.

49 Butt AA, Yan P, Bonilla H, Abou-Samra AB, Shaikh OS, Simon TG, et al. Effect of addition of statins to antiviral therapy in hepatitis $\mathrm{C}$ virus-infected persons: results from ERCHIVES. Hepatology. 2015 Aug;62(2):365-74.

50 Simon TG, Duberg AS, Aleman S, Hagstrom H, Nguyen LH, Khalili H, et al. Lipophilic statins and risk for hepatocellular carcinoma and death in patients with chronic viral hepatitis: results from a nationwide Swedish population. Ann Intern Med. 2019 Sep 3;171(5):318-27.

51 Chang KC, Hung CH, Lu SN, Wang JH, Lee $\mathrm{CM}$, Chen $\mathrm{CH}$, et al. A novel predictive score for hepatocellular carcinoma development in patients with chronic hepatitis $\mathrm{C}$ after sustained response to pegylated interferon and ribavirin combination therapy. J Antimicrob Chemother. 2012 Nov;67(11):2766-72.

52 Ioannou GN, Beste LA, Green PK, Singal AG, Tapper EB, Waljee AK, et al. Increased risk for hepatocellular carcinoma persists up to 10 years after HCV eradication in patients with baseline cirrhosis or high FIB-4 scores. Gastroenterology. 2019 Nov; 157(5):1264-78.e4.

53 Obesity: preventing and managing the global epidemic. Report of a WHO consultation. World Health Organ Tech Rep Ser. 2000; 894(i-xii):1-253.

54 Appropriate body-mass index for Asian populations and its implications for policy and intervention strategies. Lancet. 2004 Jan 10; 363(9403):157-63.

55 Low S, Chin MC, Ma S, Heng D, DeurenbergYap M. Rationale for redefining obesity in Asians. Ann Acad Med Singap. 2009 Jan; 38(1):66-9. 